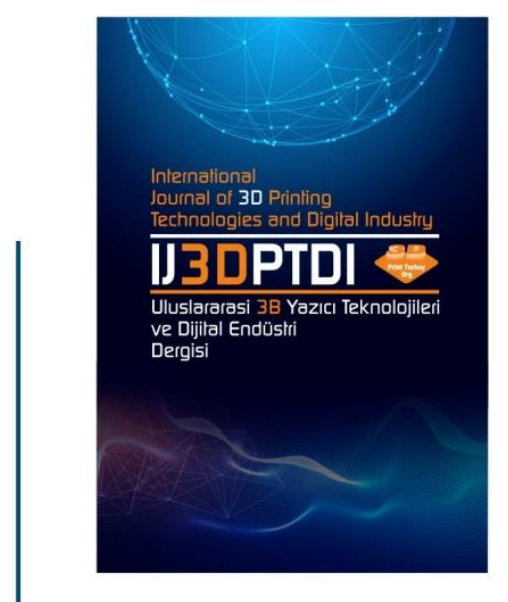

ULUSLARARASI 3B YAZICI TEKNOLOJILERI

VE DIJITAL ENDÜSTRI DERGISI

INTERNATIONAL JOURNAL OF 30 PRINTING TECHNQLOGIES AND DIGITAL INDUSTRY

I55N:2602-3350 [Online]

URL: https://dergipark.org.tr/ij3dptdi

\title{
MODAL FREQUENCY ANALYSES OF THE VARIABLE STIFFNESS MECHANISM DESIGN OF THE SOFT ROBOTIC SYSTEM
}

Yazarlar (Authors): Haydar Sahin (D)

Bu makaleye şu şekilde atıfta bulunabilirsiniz (To cite to this article): Sahin H. "Modal Frequency Analyses Of The Variable Stiffness Mechanism Design Of The Soft Robotic System" Int. J. of 3D Printing Tech. Dig. Ind., 5(3): 372-389, (2021). 


\title{
MODAL FREQUENCY ANALYSES OF THE VARIABLE STIFFNESS MECHANISM DESIGN OF THE SOFT ROBOTIC SYSTEM
}

\author{
Haydar Sahin ${ }^{a}$ \\ ȧstanbul Gedik University Engineering Faculty, Mechatronics Engineering Department, İSTANBUL, TURKEY \\ *Corresponding Author: haydar.sahin@gedik.edu.tr
}

(Received: 03.07.2021; Revised: 28.08.2021; Accepted: 22.09.2021)

\begin{abstract}
In this study, mechanism design and numerical analysis are investigated and directly simulated through additively manufacturing materials of the thermoplastic polyurethane TPU and ABS. Systematic motion planning of humanoid arm systems improved concerning the designed soft robotic arm-like variable stiffness system through the completed novel methodology for the analysis. Soft robotics variable stiffness for mechanism design is a novel research area. Additionally, the humanoid arm-like variable stiffness mechanism herein is taken as a case study in this technology. The variable stiffness types for soft robotics are inflatable robotic technology, smart materials technology, mechanism technology, and a combination of them. The variable stiffness mechanism has a novel design opportunity via the boundary conditions and the orientation of the initial conditions for soft robotics. The relation between the boundary conditions and variable stiffness is not investigated sufficiently. The novel field of study completed herein, the soft robotics variable stiffness, is a fundamental investigation for further development in the mechanism design. Variable stiffness mechanisms can transmit the translational and rotational motions into required directions with required displacements and applied forces on the multibody systems. The stiffness for the fixed-free structural constraint boundary condition of the specified initial condition orientation is $8 \mathrm{Nm} / \mathrm{rd}$ compared to the stiffness value of the $65.6 \mathrm{Nm} / \mathrm{rd}$ fixed-fixed end boundary condition.
\end{abstract}

Keywords: Soft Robotic. Variable Stiffness. Mechanism. Humanoid Robots. Modal Frequency Analysis.

\section{INTRODUCTION}

The polymeric-based materials for additive manufacturing using fusion deposition method (FDM) technology change the design of the robotics system that ought to be structured systematically for rapid mechanism prototyping. The polymer technology is providing a lightweight mechanism design with the required strength and dynamic parameters. Polymers are the preferred material for their lower density characteristics compared to steel and aluminum. Additive manufacturing technology using 4D printers is emerging to design energy-efficient and lightweight humanoid soft robotic arm-like variable stiffness mechanisms. Humanoid robotic arms are designed the closed-loop control algorithms with gravity compensations [1].

FDM technology consumes the filaments like ABS, PLA, or TPU with varied material and mechanical qualifications. Additionally, more composite materials construct within the filaments for the FDM technology. The manufacturing process for the mechanisms progresses with the technology of the $3 \mathrm{~d}$ printers. Therefore, the FDM printer manufactures them seamlessly only with the design in the CAD [2, 3]. The soft material of TPU and relatively rigid material ABS are used within the design and analysis process in the study conducted by this article. The design process involves modal frequency analysis (MFA).

The compact motors of the $4 \mathrm{D}$ printer or piezo-motors can be integrated into the joints to change the orientation of the mechanisms to function as soft robotics variable stiffness mechanisms. The humanoid arm functions as a varied stiffness mechanism structure to hold the parts with changing stiffness. The 
applied forces on the joints and the linkages are analyzed using the MBD toolbox of the Modelica ${ }^{\circledR}$ [4]. The Autodesk ${ }^{\circledR}$ Fusion 360TM Nastran ${ }^{\circledR}$ MFA toolbox of the Fusion $360^{\mathrm{TM}}$ Nastran $^{\circledR}$ or modal analysis designed with systematic boundary conditions convenient for the humanoid arm-like soft robotic variable stiffness mechanism.

Systematic motion planning for the humanoid robotic arms can be improved using the analysis results for the mechanisms to carry the dynamic forces. Therefore, these varied forces to support ought to be analyzed using the scientific methods of the MFA. Vibration analyses for the industrial robots through MFA detect the dynamic deformations due to dynamic forces [5]. However, having designed humanoid soft robotic arm-like mechanisms, these dynamic forces are prevented and minimized using lightweight and soft materials with a high damping coefficient.

The grasping with soft fingertips is analyzed to increase the grasp stability [5]. Exoskeleton robot for rehabilitation designed so that robot allows moving freely without resistance [6]. In this respect, the freefree boundary condition of the robotic arm models to test constraint of such cases here in the following sections for MFA. A lightweight robot for the end effectors design concerning a flexible robot [7]. Robot arms used for service application in the human environment are designed with vibration damping control [8]. The concurrent adaptation of the humanoid robots into the design progresses with the material and manufacturing technologies.

Human-robot collaboration is increasing with the collaborative robots integrated for the Industry 4.0 concept. Analyzing the humanoid robots for energy-efficient motions such as sitting down and standing up is crucial in realizing the state-of-the-art humanoid robots [2]. In this respect, humanoid robot arm design requires the MFA to establish the realized novel humanoid robot designs dynamically. Rehabilitation robots are developed as humanoid robots to enhance the rehabilitation of humans [9].

The motion planning strategy for the humanoid arms specifies the optimized motion, minimal effort, maneuverability, obstacle avoidance, and joint motion limits [10]. This approach in design requirements can strategically shape the humanoid soft robotic arm-like mechanism. Optimized motion and minimal effort are possible using the soft and lightweight material of TPU, which manufacture using the cost-effective and time-efficient process of FDM technology.

Soft robotics variable stiffness mechanisms have the potential to be adjusted for the requested stiffness values in the humanoid arm-like mechanism systems. Additive manufacturing technology has the potential for designing the state of the art mechanisms. Mechanisms with more linkages and joints can increase the ranges and precision of the stiffness values available to be used in robotic arm-like systems. However, the case study of three linkages with three joints is perceived for its simplicity and similarities with the humanoid arm mechanism structure. Variable stiffness structure designs applying smart materials and conductive polyurethane through position feedback [11]. Rehabilitation robots for impaired hand function are investigated for the soft haptic interface of pneumatically actuated robots [12].

Variable stiffness technologies for soft robotics systems are classified as inflatable robotic system technology, the combination of various technologies, smart materials technologies using 4D printers, and mechanism design technologies, as seen in Figure 1. The novelty of the concept is based on the mechanism design technology through the parameters of the orientations and joint boundary conditions to obtain the requested stiffness. The soft robotics variable stiffness mechanism can achieve this purpose with the study developed herein. The basic conceptual idea about the variable stiffness mechanism combines the advantages of the parallel redundant mechanisms and variable impedance actuators [4]. Variable impedance actuators control the stiffness of the joint [4]. Passive structural stiffness is designed here mainly for the serial arm-like mechanism of the humanoid soft robotic system. The fixed-fixed end of the mechanism is the structure of the parallel mechanism, which concludes to have higher stiffness than the serial mechanism 
in the results section of this study. Serial mechanisms are distinguished to have lower structural stiffness than parallel mechanisms [4].

Soft robotics variable stiffness mechanisms can be designed to structure in a series, parallel or unique stiffness patterns through the joints, boundary conditions, and orientations. The linkage type of revolute with the boundary conditions of fixed-free can affect the equivalent stiffness values for the varying orientations in the results section. The result of the study herein is conducted concerning the three joints and linkages of mechanisms. The transmission characteristics of the force and moment can be arranged by manipulating the stiffness pattern of conduction. Series elastic actuator configurations are generalized and compared [11].

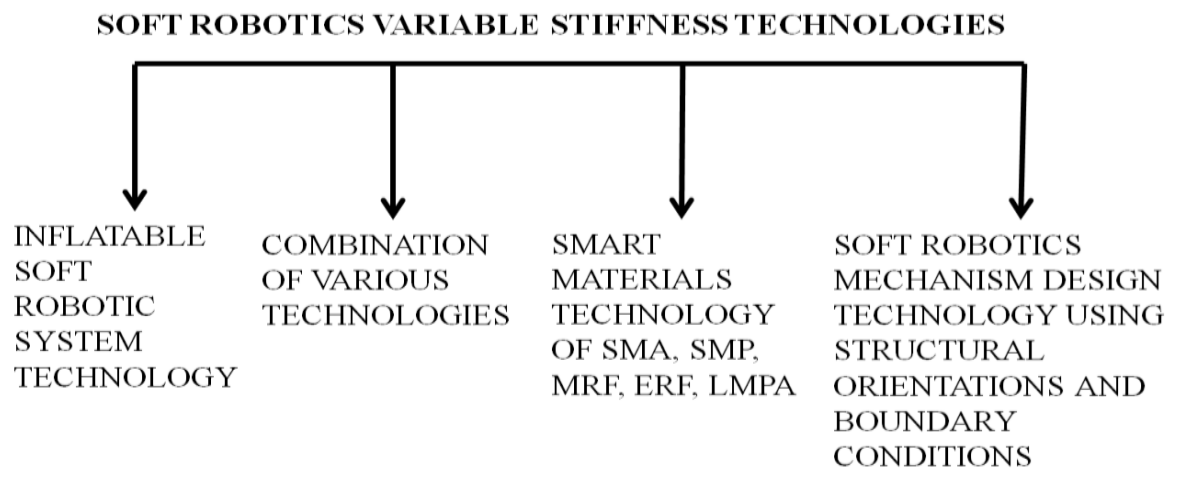

Shape memory alloy (SMA), thermoplastics (including shape memory polimer)

magnetorheological fluids (MRF), electrorheological fluids (ERF), low melting point alloy (LMPA)

Figure 1. Schematic of the variable stiffness technologies for soft robotics. The mechanism design technology to vary boundary conditions and the orientations real time to obtain requested stiffness with requested direction of the path for the transmission of force and moment.

Robotic systems require systematic analysis for dynamic forces through the MBD, FEM, and modal frequency analysis. Design and analysis of the dynamic forces for the robotic system are not complete without considering systematic MBD, FEM, and modal frequency analysis. Soft robotic mechanisms with the applied torques on the joints have higher DOF than the rigid mechanisms. Hyperelastic characteristics of the TPU material were analyzed to apply the nonlinear FEM modeling methodology of the MooneyRivlin [13] for the FEM analyses, as seen in Figure 2.

Robot systems are classified mainly as industrial robots and service robots. Additionally, service robot types are domestic robots, entertainment robots, hazardous environment robots, construction robots, and medical purpose robots [14]. Humanoid robots perform many tasks of the service robots. The locomotion obtains alternative modes by deformation induction and vibration induction as the fundamental of the soft robot locomotion [15].

Lagrange's method in driving the equations of motion for complex and many DOF systems using energy equations is more effective and efficient than Newton's method [16]. The modal frequency analysis (MFA) analytically solved is utilizing the theory of the Euler-Bernoulli using the boundary conditions mainly as the constraints of the free-free, fixed-free, fixed-fixed for the bending motion of each linkage as a beam to solve $[17,18,19]$. The system of many links of the mechanisms with the boundary conditions using the numerical Modal Frequency Analysis (MFA) can be modeled and solved through the state space modeling of global stiffness matrix, global mass matrix, global damping matrix, and global load vectors with the 
eigenvalue theory in Autodesk ${ }^{\circledR}$ Nastran $^{\circledR}$ of Fusion $360^{\mathrm{TM}}$. The compliant mechanisms obtain a higher performance when compared to the rigid joints with an extended application range $[19,20]$.

The contributions of this research study are as follows.

1. Systematic novel process developed for the soft-arm-like humanoid robot design and analysis.

2. The equivalent soft robotic variable stiffness mechanism design and analysis for humanoid soft robot arm-like system are investigated, which can be enabled by the small piezo-motors or 4D additive manufacturing technology.

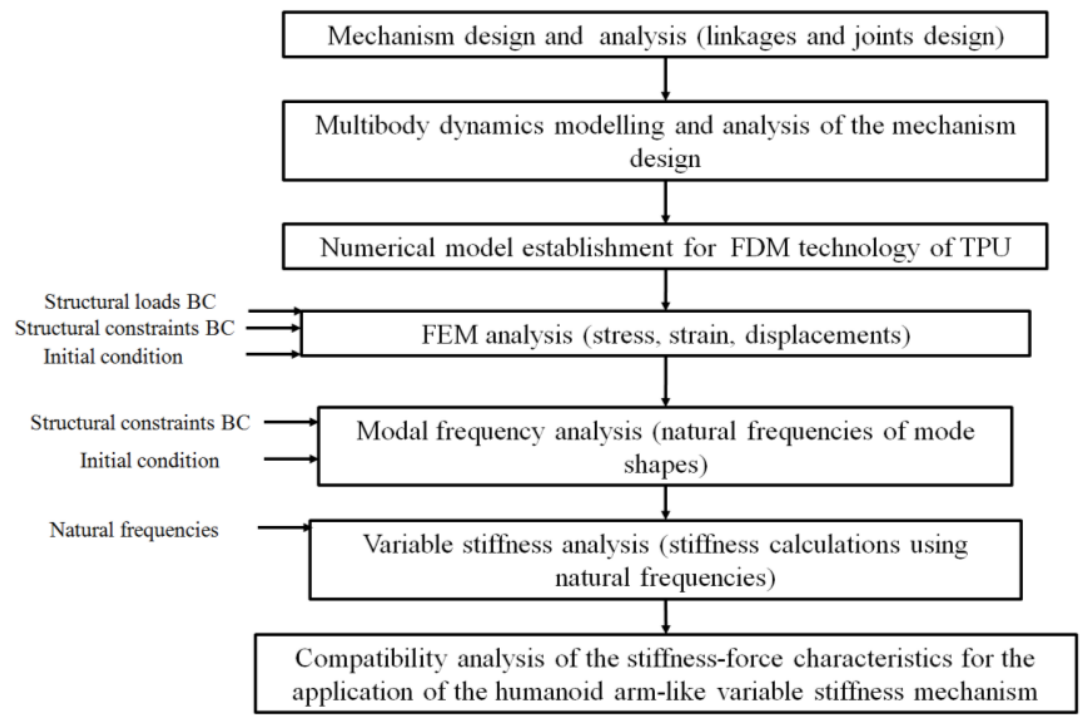

Figure 2. Schematic of the variable stiffness arm-like mechanism design and analysis for the humanoid soft robotic systems using additive manufacturing material of FDM technology.

3. The human-robot arm-like mechanism were investigated with the design and analysis of the FDM technology materials of additive manufacturing since FDM technology is progressing as a cost-effective [2, 3] manufacturing method using the parametric characteristics of materials. The filaments of composite materials are developing in addition to ABS, PLA, TPU.

Applied forces on the joints can be varied for variable stiffness applications. TPU material has the potential to be used in the variable stiffness soft robotic mechanism system. Variable stiffness values are attainable using the configurations of the boundary conditions and orientations of the initial condition for the variable stiffness soft humanoid robotic arm-like mechanism system.

The combination of the ABS material as the high stiffness source and TPU material as the low stiffness source can create the unique stiffness values of the mechanism designs. MBD and vibration dynamics for the humanoid arm-like mechanism occur with three primary boundary conditions defined in configurations 1, 2, and 3, as seen in Appendix A. The initial conditions as the orientation of the mechanism are also used as a parameter to find out the stiffness values using the natural frequencies.

\section{MATERIALS, DESIGN AND ANALYSIS PROCESS}

Upon integrating the additive manufacturing technology with the mechanism designs, the soft robotics variable stiffness mechanism can produce novel structures using the MFA design and analysis process. The soft robotics materials of the TPU and the relatively rigid material of ABS, used herein in this article, are picked for their common usage in the FDM technology. However, understanding this novel design and 
analysis process is crucial before manufacturing using the flow chart of the methodology developed herein, as seen in Figure 2.

Having designed the humanoid soft robotic components of the variable stiffness arm-like mechanism, the applied dynamics forces on the parts and joints unveiled through the strength and modal frequency analysis. A soft structure for the humanoid robotic system provides safety in interacting with humans and variable stiffness capability of motion with many DOF. Following subsections of the 3D CAD design of the mechanism with material parameters and modal frequency analysis are completed with detailed explanations.

\section{The 3D CAD Design of the Mechanism with Material Parameters}

The CAD drawing specifies, as seen for the mechanism with revolute joints in Figure 3, the qualifications defined for the TPU material. Arm in typical humanoid robots is composed of a three-bar linkage of the open-loop mechanism. The mechanism defines the linkage depicted as L and joints as J. The CAD software of Autodesk Fusion 360 is applied to determine the geometry, weight, inertia, and COG for FEM and MFA.
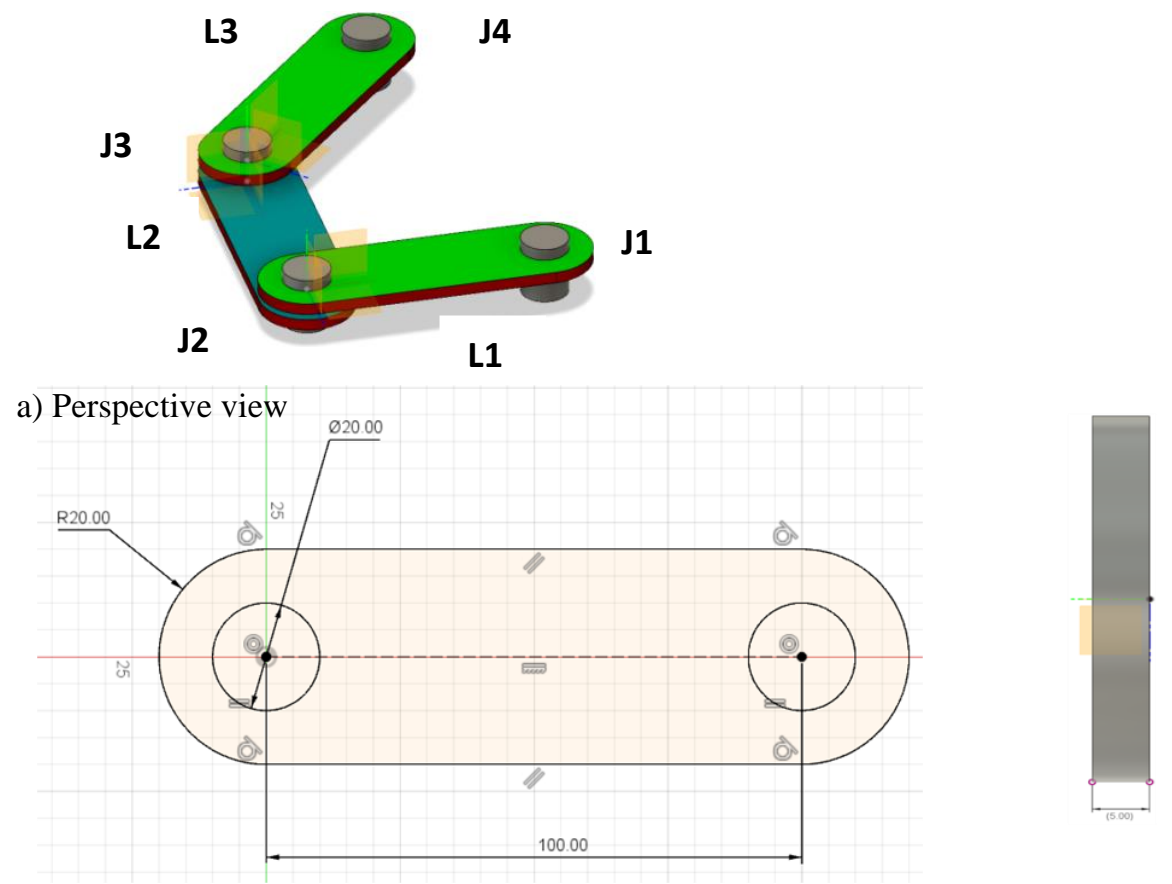

b) Front view

c) Side view with $5 \mathrm{~mm}$ thickness

Figure 3. Humanoid soft robotic arm-like mechanism design.

Boundary conditions are classified the structural constraints and structural loads. The summarized structural constraints explain the various configurations concerning FEM and MFA analysis in Table 1. The details of the boundary conditions explain the structural loads, as in Appendix A. The geometrical parameters defined in the CAD design of the mechanism with material qualifications contain the MBD modeling of mechanism design as geometry, weight, inertia, and COG, which is the subject of the following subsection. 
Table 1. Overall summary about the structural constraint boundary conditions.

\begin{tabular}{|l|l|l|}
\hline Qualifications & FEM Analysis & Modal Frequency Analysis \\
\hline Configuration 1 & J1 on L1fixed and J4 on L3 fixed & J1 on L1fixed and J4 on L3 fixed \\
\hline Configuration 2 & J1 on L1 fixed and J3 on L2 fixed & J1 on L1 free and J4 on L3 free \\
\hline Configuration 3 & & J1 on L1 fixed and J4 on L3 fixed \\
\hline
\end{tabular}

\section{Modal Frequency Analysis}

Robotic mechanisms can be excited at the natural frequencies to cause the failures and performance deteriorations if not managed conveniently. The modal frequency analysis is one of the primary vibration analyses to determine mode shapes with the natural frequencies of the mechanisms. The types of excitements imposed can be force, acceleration, or displacements with a damping ratio of 0.6 to withstand and prevent high amplitudes when the resonance situations occur. Elongation, twisting, contraction, bending, or combinations of these motions are mode shapes types with corresponding natural frequencies for the modal frequency analysis. The classified modes are fundamental, rigid-body, and harmonic.

The mode shapes and natural frequencies can be functions of the mass distributions, the stiffness of the structure, the way of the applied loads, and the geometry, as shown by Equation (1) [19] and Equation (2) [19]. Parameter values for the MFA mesh are in Table 2. The typical results of the first modes for configurations 1, 2, and 3 of the MFA are in Figure 4, explained in detail in the results section. Vibration analysis can be structured analytically using the Euler-Bernoulli equation for the beams with fixed-free end boundary conditions. Equation (1) [19] has elasticity modulus E, inertia I, the length of the linkage L, and the mass of the linkage $m$ for representation of the natural frequency $\omega_{n}$.

Table 2. Parameter values for details of MFA mesh.

\begin{tabular}{|l|l|l|}
\hline Type & Nodes & Elements \\
\hline Solids & 3268 & 1567 \\
\hline
\end{tabular}

The $\mathrm{n}^{\text {th }}$ mode natural frequency can be derived for a varied length of $\mathrm{x}$ variable using the mode shape function Equation (2) [19]. The detailed results for the MFA analysis are in the following section.

$$
\begin{aligned}
& \omega_{n}=(n \pi)^{2} \sqrt{\frac{E I}{m L^{4}}} \\
& \phi_{n}(x)=\sin \frac{n \pi}{L} x
\end{aligned}
$$

MBD and vibrational dynamics for the humanoid arm-like mechanism occur with three primary boundary conditions defined in configurations 1,2 , and 3 . These boundary conditions of the mechanism are defined as fixed - free ends, free-free, two ends fixed, respectively. The first modes of configurations of 1, 2, and 3 are shown in Figure 4 with varied boundary conditions. The boundary conditions of configuration 1, with a fixed-free end, have a higher amplitude of $3^{\text {rd }}$ linkage vibration than the other joints. The natural frequencies for the first modes of configuration 1,2, and 3 are $2.11 \mathrm{~Hz}$, translational motion with no vibration, and $5.78 \mathrm{~Hz}$, respectively. 

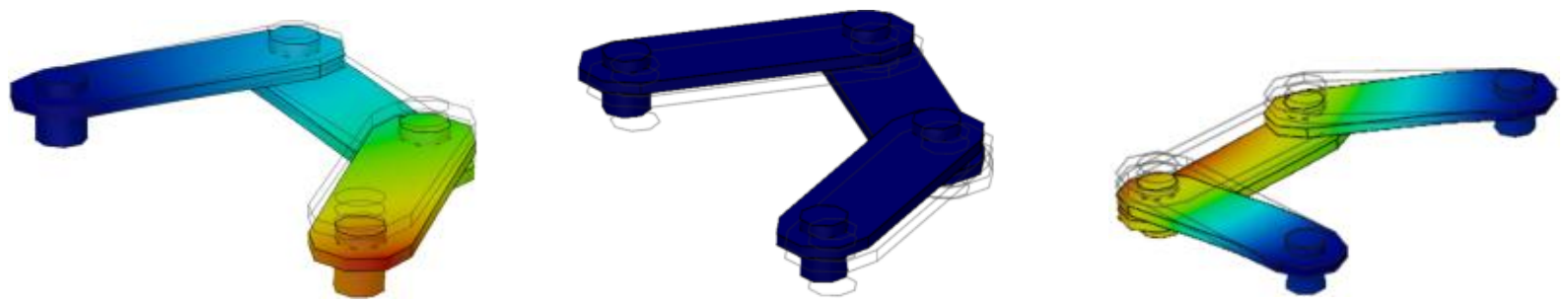

a) First mode shape for configuration 1 b) First mode shape for configuration 2 c) First mode shape for configuration 3

Figure 4. The MFA analysis for the first modes of the configuration 1,2 and 3.

Autodesk ${ }^{\circledR}$ Fusion $360^{\mathrm{TM}}$ of Nastran ${ }^{\circledR}$ programmed for the analysis of MFA for Figure 4 . The modal frequency analyses contain the boundary conditions of the free-free, fixed-free, and fixed-fixed. Appendix A explains the boundary conditions in detail.

\section{RESULTS}

The results for the FEM and MFA are completed in the following subsections.

\section{Modal Frequency Analysis Results}

Modal frequency analysis results are classified for configurations 1, 2, and 3, as seen in Table 3 with the scenarios of the 1-6. The boundary conditions are defined in the configurations of 1-2-3 when the humanoid robot arms are carrying a load. Upon defining the various stiffness applications of boundary conditions on each end as fixed-free, free-free, or fixed-fixed ends, they occur in rehabilitation or collaborative robotic applications. In the case of variable stiffness soft robotics actuators applications, the soft robotic structures should be reinforced to increase the durability in withstanding the applied forces on the system. Additionally, the damping coefficients are arranged to reduce the magnitude of the resonant effects of the natural frequency.

The major participants in the modal frequency analysis results have the $\mathrm{Y}$ component in Mode $1 \mathrm{of} 2.11 \mathrm{~Hz}$, $\mathrm{X}$ component in Mode 5 of $15.6 \mathrm{~Hz}$, and Z component in Mode 3 of $7.14 \mathrm{~Hz}$ for configuration 1, as seen in scenario 1 of Table 3. The $X$ component in Mode 1 of translational motion, Y component in Mode 2 of translational motion, and $\mathrm{Z}$ component in Mode 3 of translational motion has participated in the modal frequency analysis result for configuration 2 with natural frequencies, as seen in scenario 2 of Table 3 .

One can observe that when the stiffness of the structure with two fixed ends of configuration 3 increases the modal frequency values compared to configuration 1 of one end fixed boundary condition. This result can be observed clearly from scenario 3 of Table 8 results of configuration 3 compared to scenario 1 of Table 3. The Y component in Mode 1 of $5.78 \mathrm{~Hz}, \mathrm{X}$ component in Mode 4 of $36.79 \mathrm{~Hz}$, and Z component in Mode 5 of $41.14 \mathrm{~Hz}$ have principle participation in the modal frequency analysis result for configuration 1 , as seen in scenario 3 of Table 8. The Y component in Mode 1 of $27.26 \mathrm{~Hz}, \mathrm{X}$ component in Mode $3 \mathrm{of} 42.41 \mathrm{~Hz}$, and $\mathrm{Z}$ component in Mode 5 of $213.5 \mathrm{~Hz}$ have principle participation in the modal frequency analysis result for configuration 1 of ABS material as seen in scenario 4 of Table 3. These natural frequency values for ABS material are ten times higher frequency values than the TPU material for configuration 1 of scenario 1 in Table 3. The MFA results are shown of configurations 1 and 3 concerning the eight mode shapes, as seen in Figure 5. 
Table 3. Natural frequency results of the various configurations and orientations.

\begin{tabular}{|c|c|c|c|c|c|}
\hline \multirow{2}{*}{$\begin{array}{l}\text { Materials } \\
/ \\
\text { Scenarios }\end{array}$} & \multirow{2}{*}{$\begin{array}{l}\text { ICs Figure } \\
\text { A3 } \\
\text { Orientations }\end{array}$} & \multirow{2}{*}{$\begin{array}{l}\text { Struc. BCs in Figure } \\
\text { A2 } \\
\text { Constraints } \\
\end{array}$} & \multicolumn{3}{|c|}{ Natural Frequency $(\mathrm{Hz})$} \\
\hline & & & $\omega_{\mathrm{nX}}(\mathbf{X})$ & $\omega_{n Y}(Y)$ & $\omega_{\mathrm{nz}}(\mathrm{Z})$ \\
\hline TPU / 1 & 1 & Conf-1 & 15.6 & 2.114 & 7.14 \\
\hline TPU / 2 & 1 & Conf-2 & 0 & 0 & 0 \\
\hline TPU / 3 & 1 & Conf-3 & 41.14 & 5.787 & 83.65 \\
\hline ABS / 4 & 1 & Conf-1 & 213.5 & 27.26 & 97.56 \\
\hline TPU / 5 & 2 & Conf-2 & 0 & 0 & 0 \\
\hline TPU / 6 & 2 & Conf-1 & 25.5 & 2.787 & 7.84 \\
\hline
\end{tabular}

Variable stiffness values can be attainable by varying the structural boundary conditions shown by scenarios one and 3 of Table 3. Equation (3) can explicitly state that the stiffness is proportional to the square of the natural frequency. Therefore, the stiffness changes by the varying of the structural boundary conditions of constraints. The MFA simulation results depict the color code of the motions for scenarios 1 and 3 in Figure 5. Moreover, variable stiffness values can be attainable by varying the orientation, which is shown in scenario 6 of Table 3 and Figure 6 for configuration 1 of MFA. If compared with scenario 1 in Table 3 for varied orientation of initial condition one, the natural frequency values are changed for initial condition two as seen in scenario 6 of Table 3 .

Equation (3) can explicitly state that the stiffness is proportional to the square of the natural frequency. Therefore, the stiffness varied the change of the orientation used as an initial condition.

The variable stiffness for the arm structures of the humanoid robots is applied for collaborative robots and haptics purposes. Artificial intelligence applications with teachable robots should be designed through modal frequency analysis and finite element analysis. Lower natural frequency values for mode one will cause lower stiffness, as seen in Equation (3) [17, 19]. Configuration 1 MFA has low values of natural frequencies than configuration 3. Therefore, the stiffness values for configuration 3 are higher than configuration 1 .

$K_{x}=I_{x} \cdot \omega_{n}^{2}$

One can use these varied configurations with various BC to design variable stiffness humanoid arms for collaborative robots and haptics. The design and manufacturing process using 4D additive manufacturing technology is progressing to manufacture cost-effective mechanisms with motors integrated into the joints. The simplified stiffness calculation is completed for configuration 1 of scenario 1 using Table 3 as an example. The inertia values of $I_{x}, I_{y}, I_{z}$ are $8 \times 10^{-4} \mathrm{~kg} \cdot \mathrm{m}^{2}, 7 \times 10^{-4} \mathrm{~kg} \cdot \mathrm{m}^{2}, 6 \times 10^{-4} \mathrm{~kg} \cdot \mathrm{m}^{2}$. 


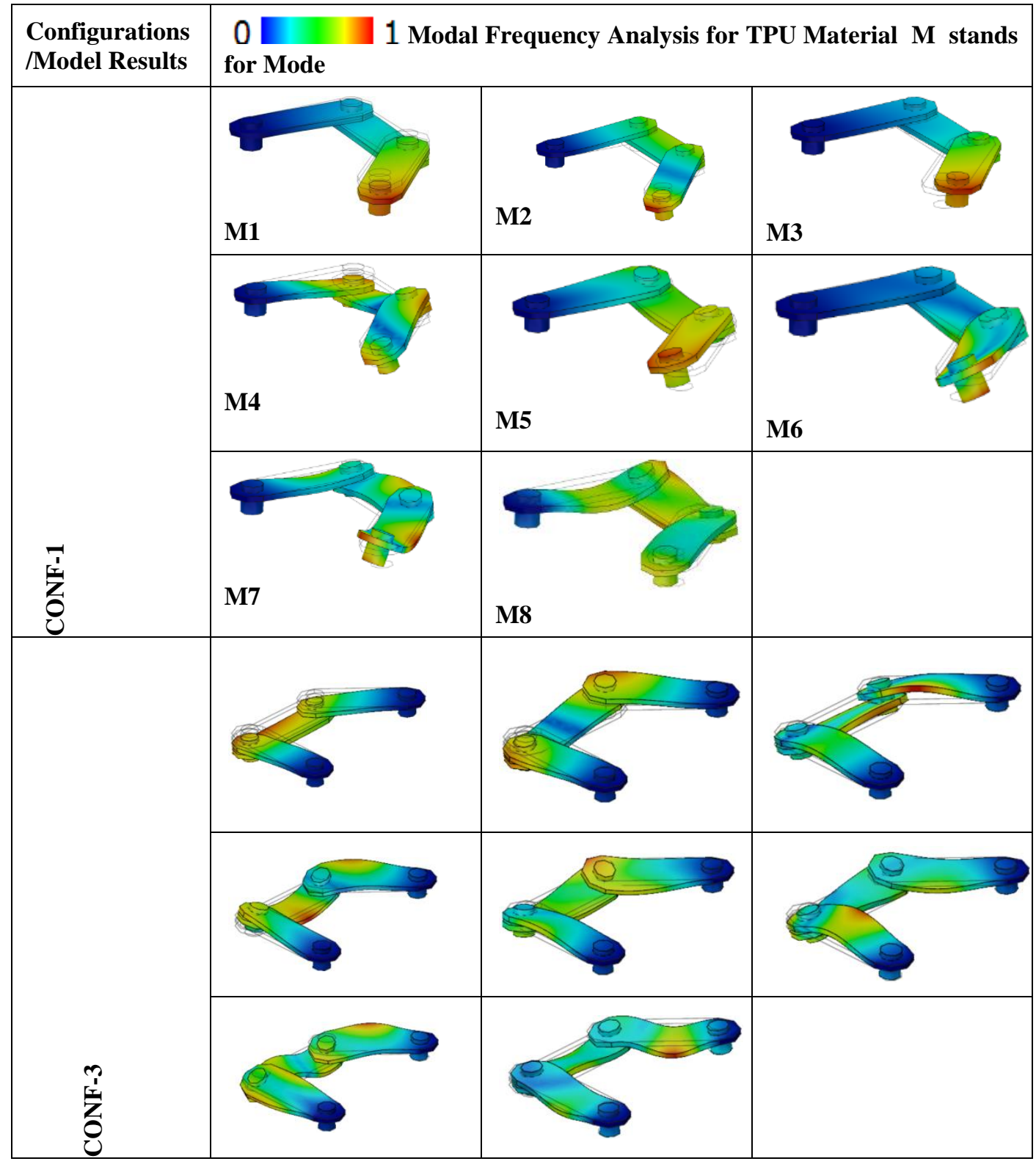

Figure 5. The MFA analysis result for initial condition 1 as varied orientation of the configuration 1 and 3 using TPU material.

The results of the Equation 3 is calculated as in the Equations of the 4 to 6

$$
\begin{array}{ll}
K_{x}=8 \times 10^{-4} \times(15.6 \times 2 \times 3.14)^{2} & =7.7 \mathrm{Nm} / \mathrm{rd}, \\
K_{y}=7 \times 10^{-4} \times(2.11 \times 2 \times 3.14)^{2} & =0.14 \mathrm{Nm} / \mathrm{rd}, \\
K_{z}=6 \times 10^{-4} \times(7.147 \times 2 \times 3.14)^{2} & =1.62 \mathrm{Nm} / \mathrm{rd},
\end{array}
$$

As a result, stiffness can be varied by both the boundary conditions and initial conditions, as stated in this subsection. The boundary conditions are stated as configurations 1, 2, and 3 for MFA. The MFA analysis 
of initial condition two is stated as varied orientation, as seen in Figure 6. TPU material is convenient to reach varied low stiffness values compared to the ABS material. Due to space limitations, the rest of the other configurations and orientations are not included here.

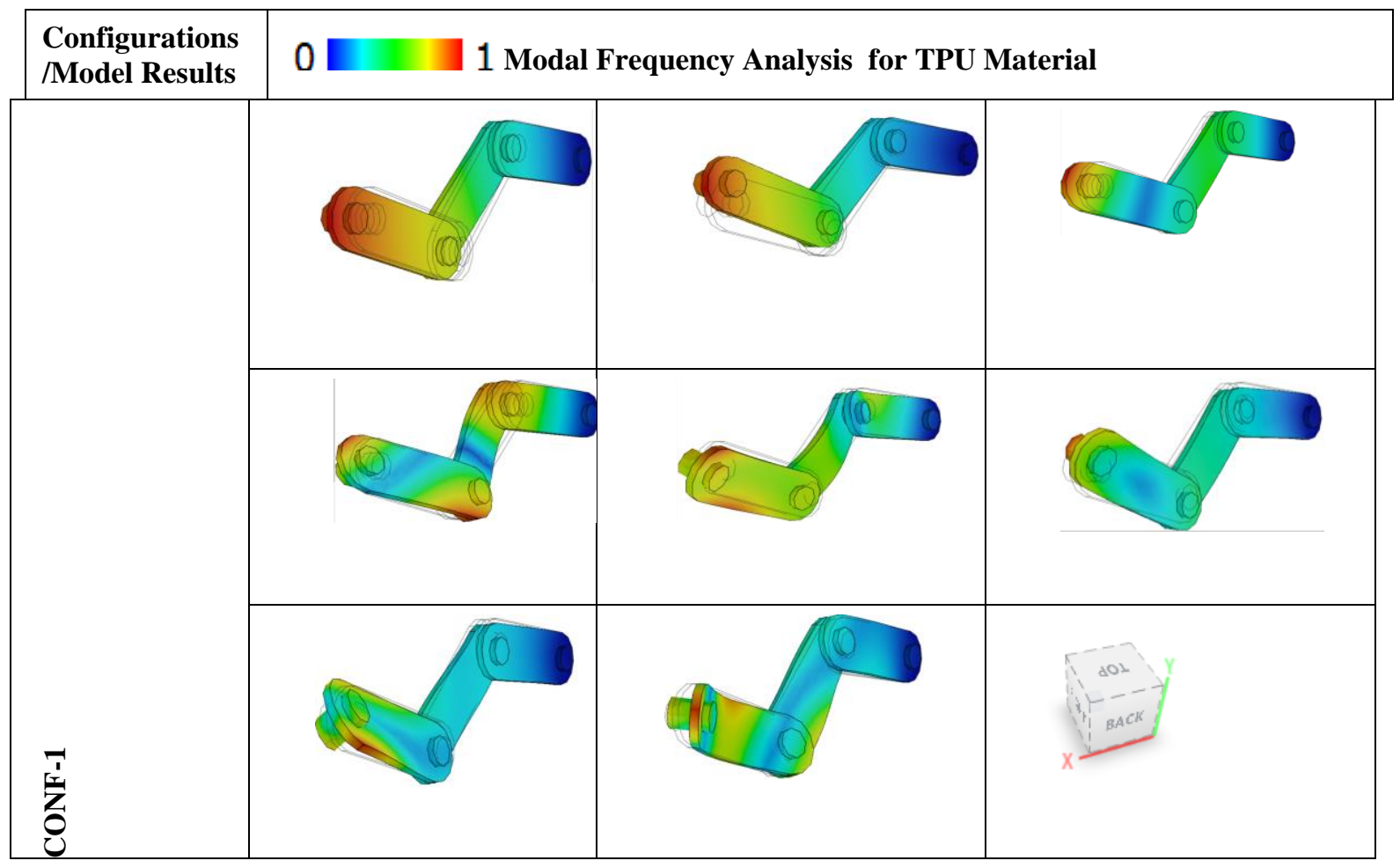

Figure 6. The MFA analysis result of initial condition 2 as varied orientation of the mechanism for the configuration 1 using TPU material.

\section{DISCUSSION}

Variable stiffness open-loop mechanism design for humanoid robots using the material of the FDM technology is not thoroughly investigated in the literature. Variable stiffness control for the humanoid robot arm systems can be achieved using the TPU material for soft robotics systems. The design of the soft robotic variable stiffness mechanism of the humanoid robot arm-like system is completed with results promising for further studies in this field here. This design can be used for humanoid robots as a haptic system that is reachable via artificial intelligence. The innovative approach in the system can also help the progress of collaborative robots, haptic systems, teachable systems, and rehabilitation systems.

Adaptronics systems including the sensors and control integrated into the materials can be designed using the design developed here. TPU material has the potential to be used as variable stiffness soft robotic mechanism system. Additive manufacturing of soft robotics is adding value with the innovations of the variable stiffness mechanism. In this respect, the TPU as soft robotics application-related compares with the ABS material in terms of FEM and MFA results. ABS material has higher natural frequencies with a higher stiffness than the TPU material.

The design process involves the dynamic applied force analysis, FEM analysis, and modal frequency analysis with stiffness calculations, as seen in Table 4. The similar orientation of the initial condition 1 for the varied configurations results in modifiable stiffness. The stiffness values for the two ends fixed boundary condition of configuration three are higher than the fixed-free boundary condition of configuration 1 . The 
same fixed-free configuration 1 for the varied orientations of the initial conditions 1 and 2 have varied stiffness results, as seen in Table 4. The detailed discussions about the changing orientations of initial conditions are considered in the following paragraph.

Table 4. MFA result of initial condition 2 as varied orientation for configuration 1 fixed- free ends using the TPU material.

\begin{tabular}{|l|l|l|l|}
\hline & $\left.\mathbf{K}_{\mathbf{x}}(\mathbf{N m} / \mathbf{r d})\right)$ & $\mathbf{K y}(\mathbf{N m} / \mathbf{r d}))$ & $\mathbf{K}_{\mathbf{Z}}(\mathbf{N m} / \mathbf{r d})$ \\
\hline Config 1 and IC 1 & 7.7 & 0.14 & 1.62 \\
\hline Config 3 and IC 1 & 53.29579 & 0.9287 & 165.776 \\
\hline Config 1 and IC 2 & 20.036 & 0.2032 & 1.90 \\
\hline
\end{tabular}

The perpendicular $2 \mathrm{~N}$ force applies to the surface of the free end of the mechanism for comparison of the maximum stress and displacements results among the various orientations, shown in Figure A3. Ten different orientations of the initial conditions in Table 5 are tested using the MFA toolbox of Fusion 360 Nastran. The calculated $\mathrm{K}_{\mathrm{x}}$ values using Equation (7) are listed for various orientations. Orientation 2 can achieve 3 times higher stiffness than orientation 1 with the same maximum displacements and stress values on the structure. Since orientations 9 and 1 as seen in Figure A3 are in a symmetrical position, the maximum stress, displacement, and stiffness values do have not much difference in between each other.

The angle of the joint close to the fixed end boundary condition location is more effective in determining the maximum stress and displacement values than the joint. Therefore, while orientations 10 and 5 have much difference in the maximum stress and displacements, orientations 8 and 9 have similar values of the maximum stress and displacements with the same joint angles of 90 degrees. The stiffness values of 5.99$20 \mathrm{Nm} / \mathrm{rd}$ can be achieved for the varied orientations of configuration 1, around the x-axis, as seen in Table 5. This variation in the stiffness designs can prove the potential to be used in soft robotic systems as a varied stiffness source. More orientations can also be created to obtain the other stiffness values. Nowadays, the current variable stiffness structures using rigid mechanisms $[19,20]$ have been constructed with many parts of a complex system. Thus, the simplified designs of soft robotic variable stiffness systems can be potentially be used as the variable stiffness mechanisms compared to the current complex rigid mechanism variable stiffness systems. Additionally, sensitive haptic system design in low stiffness and force values can be achieved compared to the rigid humanoid mechanisms. Rehabilitation robots for impaired hand function are investigated with stiffness values of $0.5-2 \mathrm{~N} / \mathrm{mm}$ for the soft haptic interface of pneumatically actuated robots [10].

Variable stiffness of the robotic arm-like mechanisms is crucial in controlling the collaborative robots, haptic systems, and rehabilitation robots for grasping objects of various softness. Teachable service and humanoid robots of various admittance and impedance control can be achieved using the variable stiffness mechanisms. A damping ratio of 0.6 will reduce the amplitudes of the disturbance forces. The ABS material with high stiffness source and TPU material with low stiffness source for soft robotic variable stiffness mechanism design can be structured to establish composite material with unique stiffness values.

Upon comparing the stiffness values in Table 5 with the research articles in the field of the humanoid robotic arm, the mechanism establishes the compatibility analysis of the stiffness. The measured stiffness values are in the ranges of 2- $20 \mathrm{Nm} / \mathrm{rad}$ for the joints of the human arm [21]. In the research article, the measured joint torque on the elbow joint is -18 to $18 \mathrm{Nm}$ as forces and moments generated by the human arm [22]. Therefore, humanoid robotic arm convenience in terms of the applied moments and stiffness can be seen in Table 10 according to the references. The three joints with linkages for the human arm are called the trunk, upper arm, and forearm in a reference to the Ph.D. dissertation [23] which have similarities with the three joints and the three linkages mechanism developed as soft robotic variable stiffness mechanism here. The 
increase in the stiffness using the concept of adding layers was shown with a diagram in a reference [16]. Vibration damping control for the robotic arm of service application [24], series elastic actuator configurations [25], stiffness issues in variable stiffness robots [26-32] are the research subjects in the field of robotic systems stiffness. The torque analyses for the electrical motors [33] and spherical elastomeric bearings [34] are developing to support the variable stiffness technology in soft robotics .

Table 5. MFA result of various orientations of initial conditions for configuration 1 of Figure A2 fixed-free ends using the TPU material.

\begin{tabular}{|c|c|c|c|c|c|c|c|}
\hline $\begin{array}{l}\text { IC's of } \\
\text { Orientation } \\
\text { Scenarios } \\
\end{array}$ & $\begin{array}{l}\text { Max } \\
\text { Stress } \\
\text { MPa } \\
\end{array}$ & $\begin{array}{l}\text { Max } \\
\text { Displacements } \\
\text { mm }\end{array}$ & $\begin{array}{l}\mathbf{N m} / \mathbf{r d} \\
\mathbf{K}_{\mathbf{x}}\end{array}$ & \begin{tabular}{|l|} 
IC's of \\
Orientation \\
Scenarios \\
\end{tabular} & $\begin{array}{l}\text { Max Stress } \\
\text { MPa }\end{array}$ & $\begin{array}{l}\text { Max } \\
\text { Displacements } \\
\text { mm }\end{array}$ & $\begin{array}{l}\mathbf{N m} / \mathbf{r d} \\
\mathbf{K}_{\mathbf{x}}\end{array}$ \\
\hline 1 & 0.02214 & 0.08384 & 7.6781 & 6 & 0.02079 & 0.08777 & 10.0415 \\
\hline 2 & 0.02186 & 0.08284 & 20.5158 & 7 & 0.02079 & 0.08777 & 8.6418 \\
\hline 3 & 0.02187 & 0.08706 & 10.1883 & 8 & 0.02318 & 0.08851 & 5.991 \\
\hline 4 & 0.01544 & 0.008415 & 6.1574 & 9 & 0.02383 & 0.08841 & 7.7274 \\
\hline 5 & 0.02283 & 0.09291 & 7.9262 & 10 & 0.05039 & 0.04934 & 8.0568 \\
\hline
\end{tabular}

The orientation scenarios of initial conditions are identified in Figure A3.

The stiffness values obtained with the FEM and MFA analysis can be used in the various system models using stiffness matrix, as seen in Equation (7) [17], as the equation of motion that can be transformed into the state-space model to solve.

$I \ddot{\theta}+D \dot{\theta}+K \theta=\tau$

Additionally, the open-loop and closed-loop control for the mechatronic and adaptronic robotics systems can be designed using the analysis results of the state space model developed. A high bandwidth control system design needed for the minimum transient response time for the structure of the mechatronic system of the robots can be achieved using the technology of the variable stiffness mechanisms. The requested force and moment transmissions through the requested path are the purposes of the stiffness matrix in the statespace model for the robotics systems.

The stiffness, displacement, and stress results of the boundary conditions listed in Figure A1 for the initial condition orientation ten are listed in Table 6 . The varied stiffness values in Table 6 for the initial condition orientation 10 of the configuration 1 and 2 as stated in Figure A1 are higher than the stiffness value in Table 5 for configuration one as stated in Figure A2. FDM technology materials of the TPU and ABS were compared and studied here. The ABS material has higher natural frequencies with a higher stiffness than the TPU material. The MFA of the TPU material is in-depth investigated for its low frequency and low stiffness characteristics compared to the ABS material, as seen in Table 6. The soft robotics variable stiffness characteristics of the TPU material for the arm-like humanoid robots are proved with these results. The stiffness for the fixed-free structural constraint boundary condition of the initial condition orientation ten, as seen in Table 5 , is $8 \mathrm{Nm} / \mathrm{rd}$ compared to the stiffness value of the $65.6 \mathrm{Nm} / \mathrm{rd}$ fixed-fixed end boundary condition as seen in Table 6 . 
Table 6. MFA result of various orientations of initial conditions from Figure A3 for configuration 1 and configuration 2 of the structural constraint boundary conditions in Figure A1 using the TPU and ABS materials.

\begin{tabular}{|l|l|l|l|l|l|l|}
\hline Material & $\begin{array}{l}\text { ICs Figure } \\
\text { A3 }\end{array}$ & \multicolumn{2}{l|}{$\begin{array}{l}\text { Struc. BCs in Figure } \\
\text { A1 }\end{array}$} & $\begin{array}{l}\text { Max. } \\
\text { Stress }\end{array}$ & $\begin{array}{l}\text { Max. Total } \\
\text { Displacement }\end{array}$ & $\begin{array}{l}\text { Stiffness } \\
\mathbf{K}_{\mathbf{x}}\end{array}$ \\
\hline & Orientation & Constraints & Loads & MPa & mm & Nm/rd \\
\hline TPU & 10 & Conf-1 & Same & 0.0525 & 5.897 & 65.605305 \\
\hline TPU & 10 & Conf-2 & Same & 0.0548 & 1.161 & 19.845605 \\
\hline ABS & 10 & Conf-1 & Same & 0.0589 & 0.03791 & 12328.444 \\
\hline ABS & 10 & Conf-2 & Same & 0.0673 & 0.006382 & 3878.2259 \\
\hline
\end{tabular}

The initial conditions of orientations generate the variable stiffness of the soft robotics mechanisms, as seen in Figure A3. In addition, boundary conditions stated as configurations 1, 2, and 3 for MFA are common structural constraints that can be used in the mechanisms as fixed and free ends, as seen in Figure A2.

\section{CONCLUSION}

Novel soft robotics variable stiffness mechanism design and analysis process is developed for the additive manufacturing technology. The variable stiffness mechanism design is developed for the analysis process of additive manufacturing technology of novel soft robotics. The BC and IC orientations are determined in terms of the variable stiffness effects of the mechanism using the TPU and ABS materials. It is shown that the combination of the TPU and ABS materials can be used as a source of low stiffness and high stiffness in a mechanism to obtain requested variable stiffness values. Structural constraint boundary conditions of the free-free, fixed-free, and fixed-fixed are designed systematically and methodologically with the realistic structural loads' boundary conditions using multi-body dynamics modeling developed for the soft robotics variable stiffness mechanisms.

The novel scientific methodology developed for soft robotic variable stiffness mechanism here was organized using MFA for integrated mechatronics and adaptronics system design concept. TPU and ABS materials are compared for FEM and MFA. The designed robotic mechanism with ABS has almost 50-150 times higher stiffness values than the TPU material, as stated in Table 6. The stiffness values of 5.99-20 $\mathrm{N} / \mathrm{m}$ are achieved for the varied orientations of the fixed-free end of the mechanisms. Higher stiffness values of $65 \mathrm{Nm} / \mathrm{rd}$ were achieved using the fixed-fixed boundary condition for the initial condition orientation 10 of TPU material.

Since FDM technology uses filaments of various materials of composites, the manufacturing process of BC and IC-related variable stiffness systems is cost-effective for humanoid robots, collaborative robots, service robots, rehabilitation robots, and haptic systems for grasping. In addition, the soft robotic variable stiffness mechanism design using the FDM technology is found to be convenient for fast prototype studies of the variable stiffness humanoid soft robotic arm-like mechanism design. TPU and composite materials have the potential to be used as a material of variable stiffness soft robotic mechanical systems with low stiffness values, which is requested in the humanoid, collaborative, service, rehabilitation robots, and grippers. Humanoid robots arm-like soft robotic systems with variable stiffness ranges are achievable by changing the orientation and configuration boundary conditions. A damping ratio of 0.6 for the soft material of TPU will cause low amplitude in the natural frequencies, which is the vibration drawback of the excitations in the natural frequencies.

TPU used for soft robotics application is also convenient as variable stiffness humanoid arm-like mechanism design. The methodology developed can be applied to various materials for the variable mechanism design of soft robots. The design concept developed for the soft robotics variable stiffness humanoid arm-like mechanism here is cost-effective, fail-safe, and stable compared to the rigid materials with closed-loop stiffness control systems. 
The future developments can structure the additional designs, material, and varied boundary conditions not simulated herein. The 4D printer technology is emerging to establish effective soft robotics variable stiffness mechanisms. The composite materials mechanisms can also be investigated with the combinations of the fixed, free, and pinned boundary conditions to obtain the variable stiffness values without needing the closed-loop control. Furthermore, velocity, acceleration, and displacement-related boundary conditions with various soft robotics composite materials of varied geometrical designs can also be the boundary conditions for further investigation in the variable stiffness mechanism of soft robotics.

\section{REFERENCES}

1. Muscolo, G.G., Hashimoto, K., Takanishi A., Dario, P., "A comparison between two force-position controllers with gravity compensation simulated on a humanoid arm”, Journal of Robotics, Vol. 2013, Pages 1-14, 2013.

2. Dizon, J.R.C., Espera, A.H., Chen, Q., Advincula, R.C., "Mechanical characterization of 3D-printed polymer" Additive Manufacturing, Vol. 20, Pages 44-67, 2018.

3. Strong, D., Kay, M., Conner, B., Wakefield T., Manogharan G., "Hybrid manufacturing - integrating traditional manufacturers with additive manufacturing (AM) supply chain", Additive Manufacturing, Vol. 21, Pages 159-173, 2018.

4. Stoeffer C., "Conceptual design of a variable stiffness mechanism using parallel redundant actuation", MS Thesis University of Liège, Liège, 2018.

5. Fasoulas, J., Sfakiotakis, M., "Modeling and grasp stability analysis for object manipulation by soft rolling fingertips", International Journal of Humanoid Robotics, Vol. 11, Issue 3, Pages 1-30, 2014.

6. Ye, W., Li, Z., Yang, C., Chen, F, Su, C.Y., "Motion detection enhanced control of an upper limb exoskeleton robot for rehabilitation training", International Journal of Humanoid Robotics, Vol. 14, Issue 1, Pages 1-17, 2017.

7. Vu, V., Liu, Z., Thomas, M., Hazel, B., "Modal analysis of a light-weight robot with a rotating tool installed at the end effector", Proc IMechE Part C: J Mechanical Engineering Science, Vol. 231, Issue 9, Pages 1664-1676, 2017.

8. Sahu, S., Choudhury, B.B, Biswal, B.B., "A vibration nalysis of a 6 axis industrial robot using FEA", Materials Today: Proceedings. Vol. 4, Pages 2403-2410, 2017.

9. Imamura, Y., Ayusawa, K., Yoshida, E., Tanaka, T., "Evaluation framework for passive assistive device based on humanoid experiments", International Journal of Humanoid Robotics, Vol. 15, Issue 3, Pages 1-25, 2018.

10. Xu, H, Ding, X., "Human-like motion planning for a 4-DOF anthropomorphic arm based on arm's inherent characteristics", International Journal of Humanoid Robotics, Vol. 14, Issue 3, Pages 1-20, 2017.

11. Yang, Y, Chen, Y., "3D printing of smart materials for robotics with variable stiffness and position feedback", in IEEE International Conference on Advanced Intelligent Mechatronics (AIM), Munich, Germany, 2017.

12. Sebastian, F., Fu, Q., Santello, M., Polygerinos P., "Soft robotic haptic interface with variable stiffness for rehabilitation of neurologically impaired hand function", Frontiers in Robotics and AI, Vol. 4, Issue 2, Pages 1-9, 2017.

13. Dilibal, S., Sahin, H., Celik, Y., "Experimental and numerical analysis on the bending response of the geometrically gradient soft robotics actuator", Archives of Mechanics, Vol. 70, Issue 5, Pages 391-404, 2018.

14. Sahin H., Guvenc L., "Household robotics - Autonomous devices for vacuuming and lawn mowing", IEEE Control Systems, Vol. 27, Issue 2, Pages 20 - 96, 2007.

15. Calisti M, Picardi G, Laschi C. "Fundamentals of soft robot locomotion", J. R. Soc. Interface,14:1-16 2017. 
16. Meng D, She Y, Xu W, Lu, W., Liang, B. "Dynamic modeling and vibration characteristics analysis of flexiblelink and flexible-joint space manipulator", Multibody System Dynamics, Vol. 43, Issue 4, Pages 321-347, 2018.

17. Montalvão, J.M., Silva, E., "Modal Analysis and Testing”, 1-34, Vol. 363, Springer, Dordrecht, 1999.

18. Howell, L.L., "Compliant mechanisms”, A Wiley-Interscience Publication, 2002.

19. Meng, Q., "A design method for flexure-based compliant mechanisms on the basis of stiffness and stress characteristics", PhD Dissertation, Università di Bologna, Pages 1-111, 2012.

20. Kramer, S.N., Saxena, A., "A simple and accurate method for determining large deflections in compliant mechanisms subjected to end forces and moments", Transactions of the ASME, Vol. 120, Pages 392-400, 1998.

21. Piovesan, D., Pierobon, A., DiZio, P., Lackner, J.R., "Experimental measure of arm stiffness during single reaching movements with a time-frequency analysis", Journal of Neurophysiology, Vol. 110, Issue 10, Pages 2484-2496, 2013.

22. Xu, Y., Terekhov, A.V., Latash, M.L., Zatsiorsky, V.M., "Forces and moments generated by the human arm: variability and control", Experimental Brain Research, Vol. 223, Pages 159-175, 2012.

23. Xu, Y., "Forces and moments generated by the human arm: variability and control", $\mathrm{PhD}$ Dissertation, The Pennsylvania State University 1-196, 2014.

24. Dasgupta, A., Nakamura Y., "Vibration damping control of robot arm intended for service application in human environment", in 8th IEEE-RAS International Conference on Humanoid Robots (ICRA) IEEE Press: Daejeon, Korea, 2008.

25. Lee, C, Kwak, S., Kwak, J., Oh, S., "Generalization of series elastic actuator configurations and dynamic behavior comparison”, Actuators, Vol. 6, Issue 26, Pages 1-26, 2017.

26. Höppner, H., "Analysis of human intrinsic stiffness modulation and its use in variable-stiffness robots", $\mathrm{PhD}$ Dissertation, Munich Technical University, 1-133, 2015.

27. Robinson, J.M., “A compliant mechanism-based variable-stiffness joint”, MS Thesis, Brigham Young University, $1-52,2015$.

28. Erickson, D., Weber, M., Sharf, I., "Contact stiffness and damping estimation for robotic systems", The International Journal of Robotics Research, Vol. 22, Issue 1, Pages 41-57, 2003.

29. Stasse, O., Flayols, T., "An overview of humanoid robots technologies, Biomechanics of Anthropomorphic Systems”, Vol. 124, Pages 281-310, 2018.

30. Wei, Y., Chen, Y., Ren, T., Chen, Q., Yan, C., Yang, Y., Li, Y. “A novel variable stiffness robotic gripper based on integrated soft actuating and particle jamming”, Soft Robotics, Vol. 3, Issue 3, Pages 1-10, 2016.

31. White, E. L., Case, J.C., Kramer-Bottiglio, R., “A soft parallel kinematic mechanism”, Soft Robotics, Vol. 5, Issue 1, Pages 1-18, 2018.

32. Sapmaz, A.R., Dilibal, S., "Makas-mafsal mekanizma tekniği kullanılarak iki serbestlik dereceli kablolu radyal makas sistemi tasarımı ve eklemeli imalat yöntemi ile üretimi", Int. J. of 3D Printing Tech. Dig. Ind., Vol. 4, Issue 3, Pages 253-263, 2020.

33. Yavuz, İ., Minaz, M. R., Kuncan, M., "1, 1 kw'lık indüksiyon motorun oluk sayısının verime ve torka etkisinin sonlu elemanlar yöntemiyle analizi”, IETS'18 International Engineering and Technology Symposium, Batman, Turkey, 3-5 Mayis, Pages 555-560, 2018.

34. Kaya, Y , Makaracı, M , Bayraklılar, S , Kuncan, M., "GMDH sinir ağı kullanılarak elastomer tabakalarm üzerinde küresel elastomerik yatağın maksimum gerilmesinin tahmini”, Gazi Üniversitesi Mühendislik Mimarlık Fakültesi Dergisi, Vol. 36, Issue 3, Pages 1331-1346, 2021. 


\section{Appendix A. Boundary Conditions for MFA and Stiffness Calculations}

\section{A.1. Boundary Conditions for FEM Analysis}

Boundary conditions for FEM analysis are stated in Figure A1.

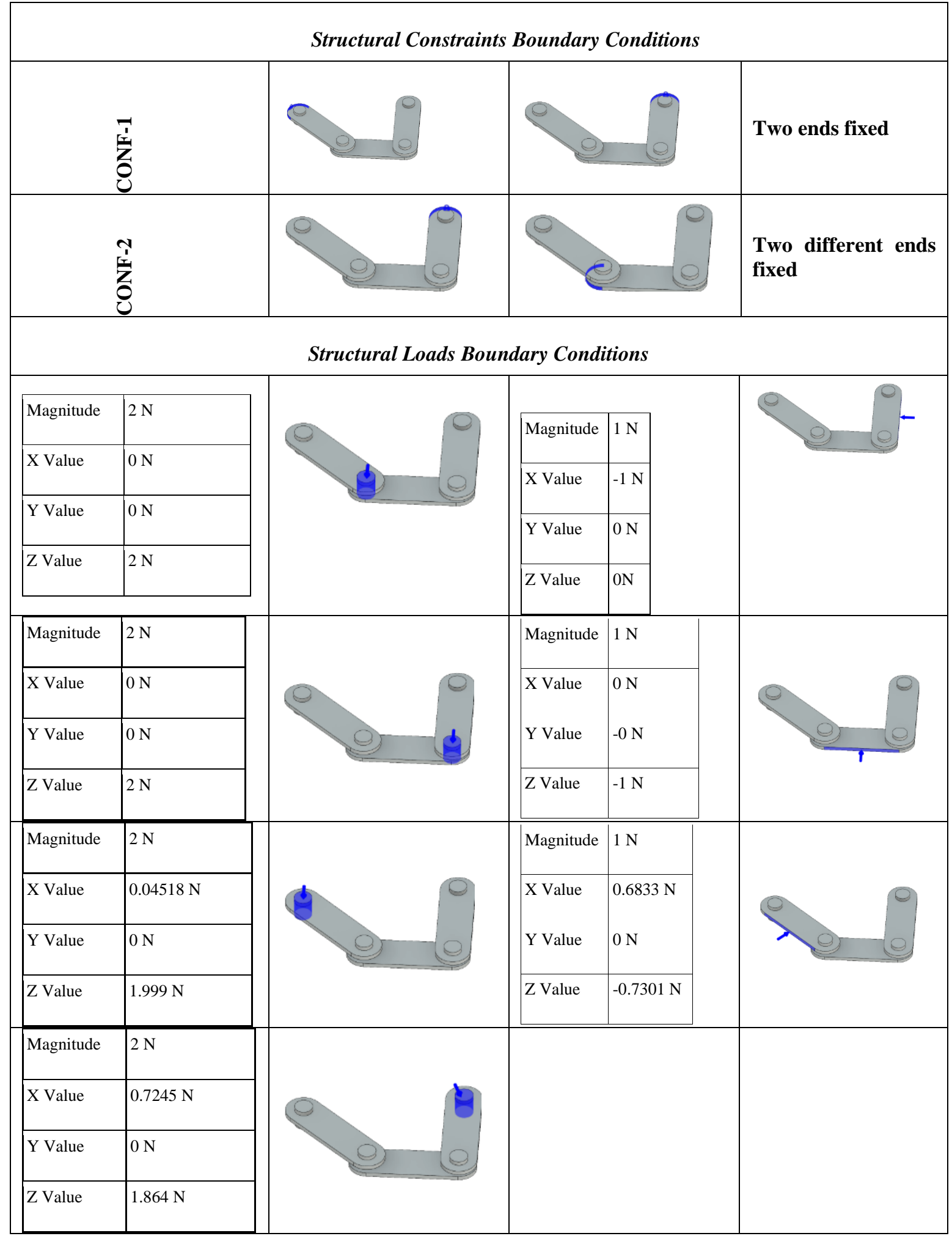

Figure A1. Boundary Conditions for FEM Analysis. 


\section{A.2. Structural Constraint Boundary Conditions for MFA}

Structural constraints boundary conditions are boundary conditions with constraints of fixed, free or pinned. The structural load boundary conditions are for applying specific value of the force, moment, velocity, acceleration. Structural loads applied FEM and FEA structures were built using Autodesk ${ }^{\circledR}$ Nastran ${ }^{\circledR}$ solver in Fusion $360^{\mathrm{TM}}$ package. Boundary conditions for FEM analysis are stated in Figure A2.

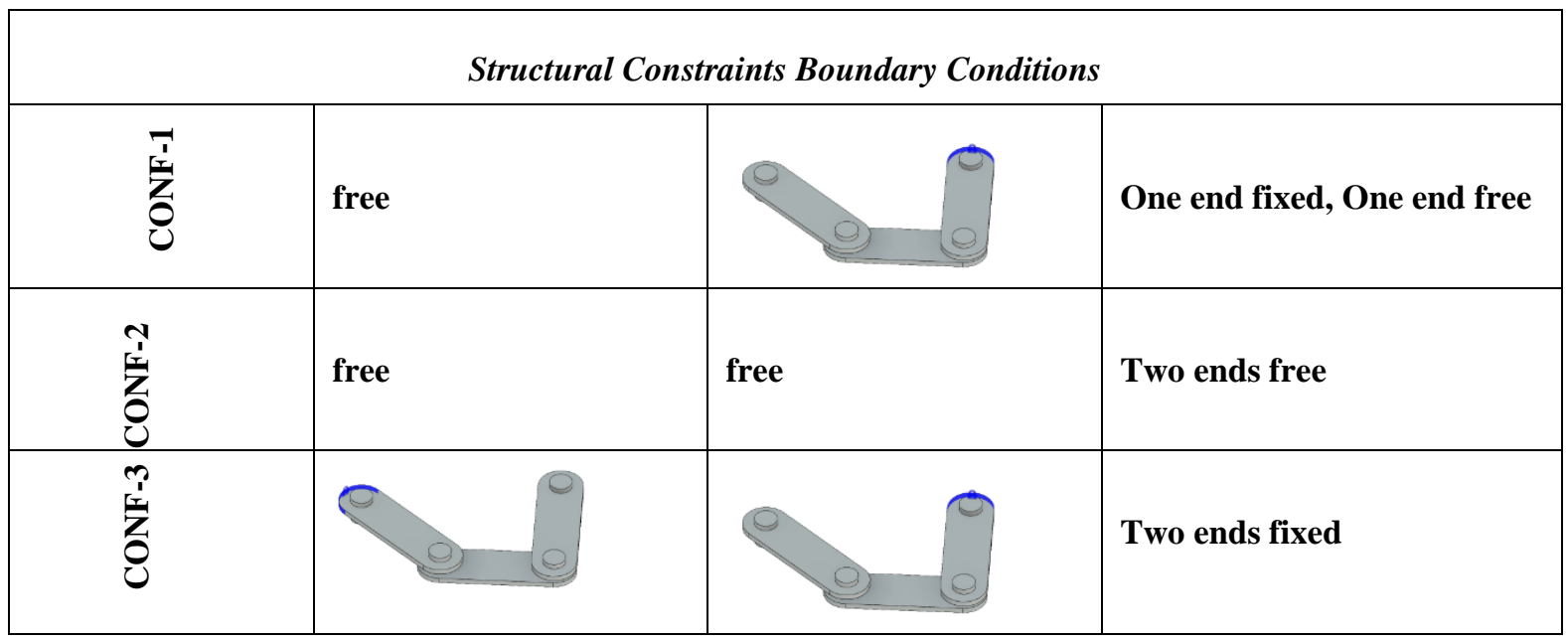

Figure A2. Boundary Conditions for MFA Analysis. 


\section{A.3. Various Orientation of the Initial Conditions of the MFA Configuration 1 (fixed-free) for the Stiffness Analysis}

Simplified depiction of the mechanisms with orientations of ICs are shown in Figure A3.

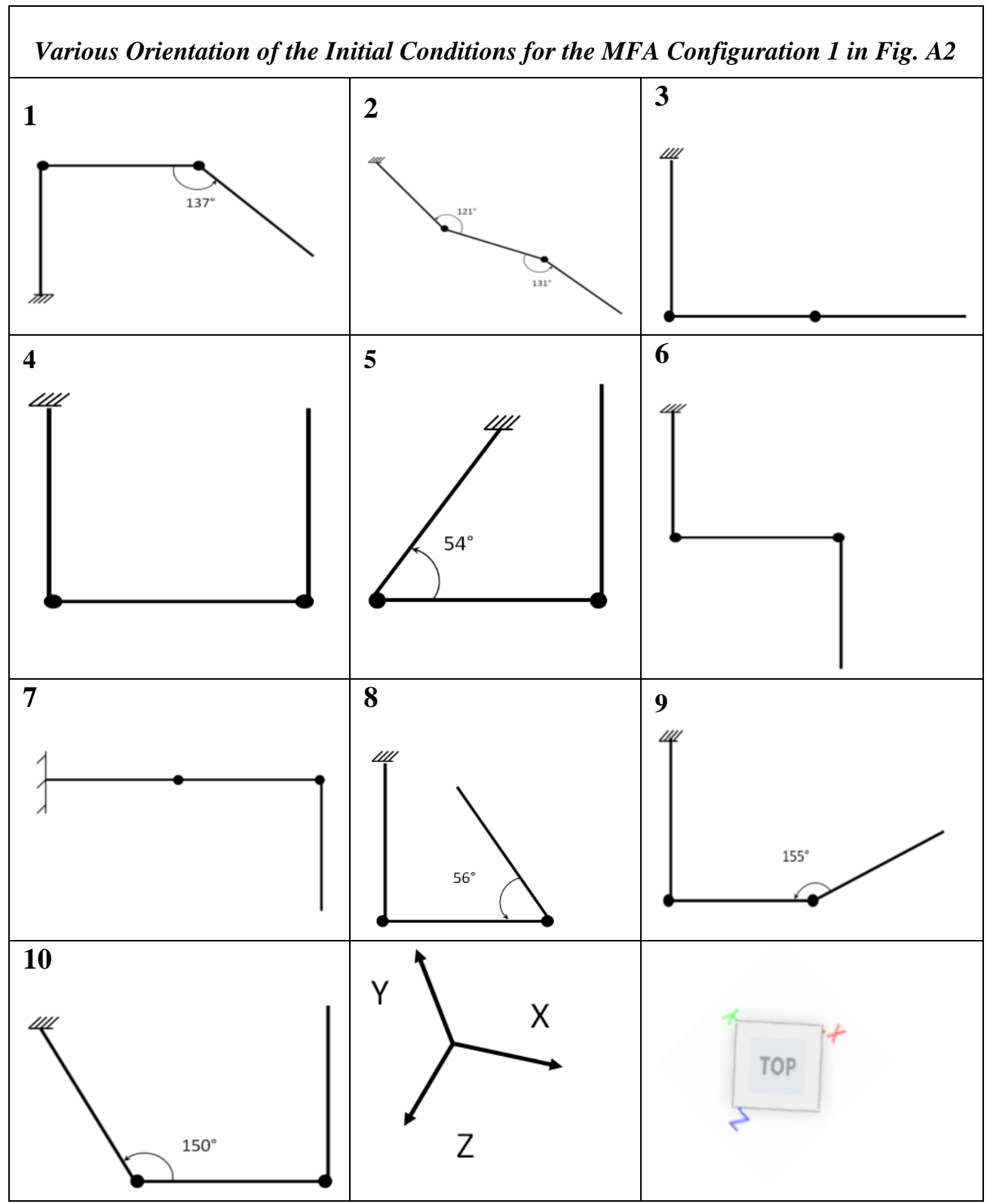

Figure A3. Various Orientation of the Initial Conditions for the MFA Configuration 1. 